\title{
Elastic Wave Motions and an Electromagnetic Field for a Photothermal Theory with Two Temperature under Hydrostatic Initial Stress
}

\author{
Khaled Lotfy ${ }^{1,2, *}$ and Wafaa Hassan ${ }^{2,3}$ \\ 1 Math. Dept., Faculty of Science, Zagazig University, Zagazig 44519, Egypt; e-mail@e-mail.com \\ 2 Math. Dept., Faculty of Science and Arts, Al-Mithnab, Qassim University 931, Buraidah 51931, Al-Mithnab, \\ Saudi Arabia ; shadzd@yahoo.com \\ 3 Math.and Phy. Dept., Faculty of Engineering, Port Said University, Port Said 42521, Egypt \\ * Correspondence: khlotfy_1@yahoo.com
}

\begin{abstract}
This paper investigates the influence of magnetic field for a two dimensional deformations on a two temperature problem at the free surface of a semi-infinite semiconducting medium under the effect of mechanical force during a photothermal theory and the effect of hydrostatic initial stress on the medium. The Harmonic Wave Method (Normal Mode Analysis) has been used to obtain the equations of elastic waves, heat conduction equation, quasi-static electric field, carrier density, two temperature coefficient, ratios, and constitutive relationships for the thermo-magnetic-electric medium. The effects of several parameters as thermoelastic and thermoelectric coupling parameters and two temperature parameter of this force on the displacement component, force stress, carrier density and temperature distribution has been depicted graphically.
\end{abstract}

Keywords: photothermal theory; carrier density; magnetic field; the harmonic wave; two temperature

\section{Introduction}

Recently, more attentions has been made for the theory of thermoelasticity because of its utilitarian aspects in diverse fields, especially, Engineering, Structures, Geology, Biology, Geophysics, Acoustics, Physics, Plasma, etc. The development of initial stress in the medium is due to many reasons such as the process of quenching, resulting from difference of temperatures, slow process of creep, differential external forces, and gravity variations. The earth is supposed to be under high initial stress. The researchers have shown much interest to study the effect of these stresses on the propagation of waves. Initial stress in solids has the significant influence on the mechanical response of the material from an initially stressed configuration and has applications in geophysics, engineering structures, and in the behavior of soft biological tissues. Initial stress arises from processes, such as manufacturing or growth and is present in the absence of applied loads. The classical uncoupled and coupled theory of thermoelasticity predicts two phenomena not compatible with physical experiments. The theory of coupled thermoelasticity introduced by Boit [1], to overcome the first shortcoming. The conventional dynamic theory of thermoelasticity (CD theory) is based on the classical Fourier heat conduction law. This theory assumes that a thermal signal propagates at infinite speeds since the resulting governing equation is parabolic in nature. Among generalized models, the Lord and Șhulman (LS) model [2], with one relaxation time, and the Green and Lindsay (GL) model [3], with two relaxation times, are familiar to many researchers and many works have been done under these theories [4-6].

Gordon et al. [7] introduced the first photothermal theory, when they found an intracavity sample where a laser-based apparatus gave rise to photothermal blooming, the photothermal lens. Kreuzer showed that photoacoustic spectroscopy could be used for sensitive analysis when laser light 
sources were utilized [8]. After that photothermal methods have been used to measure temperature, thermal diffusivities, sound velocity, bulk flow velocities, surface thickness, and specific heats [9-11]. The photothermal generation during a photothermal process was studied by many authors. For semiconductor materials, the mechanism of this process includes two parts: the propagation of a thermal wave causes elastic vibrations in the medium; this is the thermoelastic (TE) mechanism of photothermal generation.

The problem of interaction between electromagnetic field, temperature, stresses and strains in a thermoelastic solid is relevant for a number of applications such as geophysics for understanding the effect of the Earth's magnetic field on seismic waves, damping of acoustic waves in a magnetic field, emissions of electromagnetic radiations from nuclear devices, development of a highly sensitive super conducting magnetometer, electrical power engineering, optics etc. The magneto-thermoelastic disturbances generated by a thermal shock in an elastic half-space having a finite conductivity have been investigated by Puri [12]. Among the authors who considered the generalized magnetothermoelastic equations are Nayfeh and Nemat-Nasser [13] who studied the propagation of plane waves in a solid under the influence of an electromagnetic field. They obtained the governing equations in the general case and the solutions for some particular cases.

The theory of heat conduction in a deformable body, formulated by Chen et al. [14]-[16] depends on two different temperatures the conductive temperature and the thermo dynamical temperature. Chen et al. [16] discussed the difference between these two temperatures is proportional to heat supply. The existence, structural stability, convergence and spatial behavior of two temperature thermoelasticity have been provided by Quintanilla and Tien [17]. Youssef [18] has developed a new model of generalized thermoelasticity that depends on two temperatures $T$ and $\phi$, where the difference between the two temperatures is proportional to heat supply $\phi_{, i i}$ with a non-negative constant a (length ${ }^{2}$ ).

Othman and Lotfy [19] studied the plane waves in generalized thermo-microstretch elastic halfspace by using a general model of the equations of generalized thermomicrostretch for a homogeneous isotropic elastic half space. Othman et al. [20] studied the generalized thermomicrostretch elastic medium with gravitational effect and initial stress for different theories. Lotfy [21] studied Two temperature generalized magneto-thermoelastic interactions in an elastic medium under three theories. Lotfy and Hassan [22] studied Normal Mode Method for TwoTemperature Generalized Thermoelasticity under Thermal Shock Problem. Abo-Dahab et al. [23] studied generalized magneto-thermoelasticity with fractional derivative heat transfer for a rotation of a fibre-reinforced thermoelastic.

The plane waves propagation is investigated under the influence of magnetic field to solve the two temperature problem in a two dimensional deformation at the free surface of a semi-infinite semiconducting medium during a photothermal process under the effect of mechanical force as hydrostatic initial stress on the medium. The harmonic wave method was used to obtain the two temperature parameter with stress under the initial stress in the medium, exact expression of Normal displacement, Normal force stress, carrier density and temperature distribution.

\section{Basic Equations}

We consider the problem of a thermoelastic half space $(x \geq 0)$ A magnetic field with constant intensity $\vec{H}=\left(0, H_{0}, 0\right), h$ is the induced magnetic field acting parallel to the boundary plane (taken as the direction of the $y$-axis). The surface of the half-space is subjected to a thermal shock which is a function of $z$ and $t$. Thus, all quantities considered will be functions of time variable $t$, and co-ordinates $x$ and $z$. We begin our consideration with linearized of electro-dynamics slowly moving medium, the variation of magnetic field and electric field are given by Maxwell.s equations:

$$
\vec{J}=\operatorname{curl} \vec{h}-\mu_{0} \overrightarrow{\dot{E}},
$$




$$
\begin{aligned}
& \operatorname{curl} \vec{E}=-\mu_{0} \overrightarrow{\dot{H}}, \\
& \vec{E}=-\mu_{0}(\overrightarrow{\dot{u}} \times \vec{H}), \\
& \operatorname{div} \vec{H}=0,
\end{aligned}
$$

Where $\mu_{0}$ is the magnetic permeability, $\varepsilon_{0}$ is electric permeability and $\overrightarrow{\dot{u}}$ is the particle velocity of the medium, and the small effect of temperature gradient on $\vec{J}$ is also ignored. The above equations a dot denotes differentiation with respect to time. Expressing the components of the vector $\vec{J}=\left(J_{1}, J_{2}, J_{3}\right)$ (The current density vector $\vec{J}$ be parallel to $\vec{E}$, thus) in terms of displacement, where

$J_{x}=-\left(\frac{\partial h}{\partial z}+\mu_{0} H_{0} \varepsilon_{0} \ddot{w}\right), J_{z}=\frac{\partial h}{\partial x}+\mu_{0} H_{0} \varepsilon_{0} \ddot{u}$, and $J_{y}=0$, by eliminating the quantities $\vec{h}$ and $E$ from equation (1).

The components of the magnetic intensity vector in the medium are

$$
H_{x}=0 \quad, H_{z}=0, \quad H_{y}=H_{0}+h(x, y, z)
$$

The electric intensity vector is normal to both the magnetic intensity and the displacement vectors. Thus, it has the components

$$
E_{x}=-\mu_{0} H_{0} \dot{w} \quad, E_{z}=\mu_{0} H_{0} \dot{u}, \quad E_{0}=0
$$

It is assumed that the theoretical analyses of the transport process in a semiconductor contain in the consideration of coupled plasma waves, thermal waves and elastic waves simultaneously. The carrier density $\mathrm{N}(\vec{r}, \mathrm{t})$, temperature distribution $\mathrm{T}(\vec{r}, \mathrm{t})$, and elastic displacement $\mathbf{u}(\vec{r}, \mathrm{t})$ are the main variable quantities. For a medium with two temperatures of a linear, homogeneous and isotropic properties of the medium whose state can be expressed in terms of the space variables $x, z$ ( $\vec{r}$ is the position vector) and the time variable t. The coupled plasma, thermal and elastic transport equations can be given below (with new model under two temperature) as a vector form as $[5,18,24$, 25] as,

$$
\frac{\partial N(\vec{r}, t)}{\partial t}=D_{E} \nabla^{2} N(\vec{r}, t)-\frac{N(\vec{r}, t)}{\tau}+\kappa T(\vec{r}, t)
$$

Body couples, the carrier density and heat sources can be written by following the equations

$$
\rho C_{e} \frac{\partial \mathrm{T}(\vec{r}, t)}{\partial t}=k \nabla^{2} \phi(\vec{r}, t)+\frac{E_{g}}{\tau} N(\vec{r}, t)+\gamma T_{0} \nabla \cdot \frac{\partial \vec{u}(\vec{r}, t)}{\partial t} .
$$

The equations of motion and constitutive relations for a magnetic field in the absence of body forces can be written as:

$$
\begin{aligned}
& \rho \frac{\partial^{2} \vec{u}(\vec{r}, t)}{\partial t^{2}}=\left(\mu-\frac{p}{2}\right) \nabla^{2} \vec{u}(\vec{r}, t)+\left(\mu+\lambda-\frac{p}{2}\right) \nabla(\nabla \cdot \vec{u}(\vec{r}, t))-\gamma\left(1+\tau_{\theta} \frac{\partial}{\partial t}\right) \nabla \mathrm{T}(\vec{r}, t)- \\
& \delta_{n} \nabla N(\vec{r}, t)++\mu_{0}(\vec{J} \times \vec{H})
\end{aligned}
$$

where, $\kappa=\frac{\partial N_{0}}{\partial T} \frac{T}{\tau}, N_{0}$ is equillibrium carrier concentration at temperature $T$. Vasil'ev and Sandomirskii first stated that, in the case of harmonic modulation laser, the thermal activation coupling parameter $\kappa[26]$ is negligible in the case of relatively low temperature and $N_{0}$ satisfies 
the Sablicov's, Vasil'ev, and Sandomirskii inequality. Using theoretical analytical as well as experimental measurements, Christofides et al. [27] gave a precise map concerning the violation of the inequality under various conditions including modulation frequency and doping concentration. In this article, the general case, that is, the non-zero thermal activation coupling parameter was studied. $D_{E}$ is the carrier diffusion coefficient, $\tau$ is the photogenerated carrier lifetime, $E_{g}$ is the energy gap of the semiconductor. $\mu$ and $\lambda$ are the Lamé elastic constants, $\rho$ is the density, $P$ is the initial stress, $k$ is the thermal conductivity of the sample, $T_{0}$ is the absolute temperature. $\gamma=(3 \lambda+2 \mu) \alpha_{T}$ is the volume thermal expansion where $\alpha_{T}$ is the co-efficient of linear thermal expansion and $C_{e}$ is specific heat at constant strain of the solid plate, $\delta_{n}$ is the difference of deformation potential of conduction and valence band. In equation (8), the second term on the right side characterizes the effect of heat generation by the carrier volume and surface de-excitations in the sample and third term describes the heat generated by stress waves respectively. In the elastic equation (9), the third and fourth terms describes source term and influence of the thermal wave, plasma wave on the elastic wave, respectively [28]. We restrict our analysis parallel to $x z$-plane, so the displacement is defined by $\vec{u}=(u, 0, w), \quad u(x, z, t), w(x, z, t)$, also we define the strain as $e=u_{x}+w_{z}$.

The relation between the heat conduction and the dynamical heat takes the form

Error! Objects cannot be created from editing field codes.

where $a>0$ two-temperature parameter, Youssef [18].

The constitutive equation takes the form

$$
\begin{gathered}
\sigma_{\mathrm{xx}}=(2 \mu+\lambda) \frac{\partial u}{\partial x}+\lambda \frac{\partial w}{\partial z}-(3 \lambda+2 \mu)\left(\alpha_{T} T+d_{n} N\right)-P \\
\sigma_{\mathrm{zz}}=(2 \mu+\lambda) \frac{\partial w}{\partial z}+\lambda \frac{\partial u}{\partial x}-(3 \lambda+2 \mu)\left(\alpha_{T} T+d_{n} N\right)-P \\
\sigma_{\mathrm{xz}}=s_{1} \frac{\partial u}{\partial z}+s_{2} \frac{\partial w}{\partial x}
\end{gathered}
$$

Where $s_{1}=\mu+\frac{p}{2}$ and $s_{2}=\mu-\frac{p}{2}$.

\section{Formulation of the Problem}

Use the vector analysis, from (1)-(4) we note that the field equations and constitutive relations in generalized linear thermoelasticity with the effect of magnetic field and without body forces and heat sources are

$$
\begin{aligned}
\rho\left(\frac{\partial^{2} u}{\partial t^{2}}\right)= & \left(2 \mu+\lambda-\frac{p}{2}\right) \frac{\partial^{2} u}{\partial x^{2}}+\left(\mu+\lambda-\frac{p}{2}\right) \frac{\partial^{2} w}{\partial x \partial z}+\left(\mu-\frac{p}{2}\right) \frac{\partial^{2} u}{\partial z^{2}}- \\
& \gamma \frac{\partial T}{\partial x}-\delta_{n} \frac{\partial N}{\partial x}-\mu_{0} H_{0} \frac{\partial h}{\partial x}-\varepsilon_{0} \mu_{0}^{2} H_{0}^{2} \frac{\partial^{2} u}{\partial t^{2}} \\
\rho\left(\frac{\partial^{2} w}{\partial t^{2}}\right)= & \left(\mu-\frac{p}{2}\right) \frac{\partial^{2} w}{\partial x^{2}}+\left(\mu+\lambda-\frac{p}{2}\right) \frac{\partial^{2} u}{\partial x \partial z}+\left(2 \mu+\lambda-\frac{p}{2}\right) \frac{\partial^{2} w}{\partial z^{2}}-
\end{aligned}
$$




$$
\gamma \frac{\partial T}{\partial z}-\delta_{n} \frac{\partial N}{\partial z}-\mu_{0} H_{0} \frac{\partial h}{\partial z}-\varepsilon_{0} \mu_{0}^{2} H_{0}^{2} \frac{\partial^{2} w}{\partial t^{2}}
$$

For simplicity, we will use the following non-dimensional variables

$$
\begin{aligned}
\left(x^{\prime}, z^{\prime}, u^{\prime}, w^{\prime}\right) & =\frac{(x, z, u, w)}{C_{T} t^{*}}, \quad t^{\prime}=\frac{t}{t^{*}}, \quad\left(T^{\prime}, \varphi^{\prime}\right)=\frac{\gamma(T, \varphi)}{2 \mu+\lambda}, N^{\prime}=\frac{\delta_{n} N}{2 \mu+\lambda} \\
\sigma_{i j}^{\prime} & =\frac{\sigma_{i j}}{\mu}, \quad\left(\Pi^{\prime}, \psi^{\prime}\right)=\frac{(\Pi, \psi)}{\left(C_{T} t^{*}\right)^{2}}, h^{\prime}=\frac{h}{\rho C_{T}} . \\
h & =H_{0}(0, e, 0) .
\end{aligned}
$$

Where $\mathrm{e}$ is the dilatation.

Assuming the scalar potential functions $\Pi(x, z, t)$ and $\psi(x, z, t)$ defined by the relations in the non-dimensional form:

$$
u=\frac{\partial \Pi}{\partial x}+\frac{\partial \psi}{\partial z}, \quad \quad \mathrm{w}=\frac{\partial \Pi}{\partial \mathrm{z}}-\frac{\partial \psi}{\partial x} .
$$

Hence, using a scalar function and equation (16) in equations (7)-(8) and (14)-(15), we have (dropping the dashed for convenience).

$$
\begin{gathered}
\left(\nabla^{2}-q_{1}-q_{2} \frac{\partial}{\partial t}\right) N+\varepsilon_{3} T=0 \\
\nabla^{2} \phi-\frac{\partial T}{\partial t}+\varepsilon_{2} N+\varepsilon_{1} \frac{\partial}{\partial t} \nabla^{2} \Pi=0 \\
\left(\alpha \nabla^{2}-R_{H} \frac{\partial^{2}}{\partial t^{2}}\right) \Pi-T-N=0 \\
\left(\nabla^{2}-R_{H} \beta^{2} \frac{\partial^{2}}{\partial t^{2}}\right) \psi=0 \\
\varphi-T=a \nabla^{2} \varphi
\end{gathered}
$$

We can obtain from Eqs. (8) and a scalar potential functions

$$
h=\nabla^{2} \Pi
$$

where,

$$
\begin{aligned}
& q_{1}=\frac{k t^{*}}{D_{E} \rho \tau C_{e}}, \quad q_{2}=\frac{k}{D_{E} \rho C_{e}}, \quad \varepsilon_{1}=\frac{\gamma^{2} T_{0} t^{*}}{k \rho}, \varepsilon_{2}=\frac{\alpha_{T} E_{g} t^{*}}{d_{n} \rho \tau C_{e}}, \varepsilon_{3}=\frac{d_{n} k k t^{*}}{\alpha_{T} \rho C_{e} D_{E}}, \\
& C_{T}^{2}=\frac{2 \mu+\lambda-\frac{p}{2}}{\rho}, C_{L}^{2}=\frac{\mu-\frac{p}{2}}{\rho}, \beta^{2}=\frac{C_{T}^{2}}{C_{L}^{2}}, \quad \delta_{n}=(2 \mu+3 \lambda) d_{n}, \quad t^{*}=\frac{k}{\rho C_{e} C_{T}^{2}}, \\
& R_{H}=1+\varepsilon_{0} \mu_{0}^{2} H_{0}^{2} / \rho, \alpha=1+\mu_{0} H_{0}^{2} .
\end{aligned}
$$


Here, $\varepsilon_{1}$ and $\varepsilon_{3}$ represents the thermoelastic coupling parameter and the thermoelectric coupling parameter.

Stress components in non-dimensional form becomes

$$
\begin{gathered}
\sigma_{\mathrm{xx}}=\frac{(2 \mu+\lambda)}{\mu} \frac{\partial^{2} \Pi}{\partial x^{2}}+\frac{\lambda}{\mu} \frac{\partial^{2} \Pi}{\partial z^{2}}+2 \frac{\partial^{2} \psi}{\partial x \partial z}-\frac{(2 \mu+\lambda)}{\mu}(T+N)-P, \\
\sigma_{\mathrm{zz}}=\frac{(2 \mu+\lambda)}{\mu} \frac{\partial^{2} \Pi}{\partial z^{2}}+\frac{\lambda}{\mu} \frac{\partial^{2} \Pi}{\partial x^{2}}-2 \frac{\partial^{2} \psi}{\partial x \partial z}-\frac{(2 \mu+\lambda)}{\mu}(T+N)-P, \\
\sigma_{\mathrm{xz}}=\frac{s_{1}}{\mu} \frac{\partial^{2} \psi}{\partial z^{2}}+2 \frac{\partial^{2} \Pi}{\partial x \partial z}-\frac{s_{2}}{\mu} \frac{\partial^{2} \psi}{\partial x^{2}} .
\end{gathered}
$$

\section{Solution of the problem}

For a harmonic wave propagated in the direction, where the wave normal lies in the $x z$-plane, to solve the equations (9)-(13) for the physical variable, we assume the following

$$
\left[\Pi, \psi, \varphi, T, \sigma_{i j}, N\right](x, z, t)=\left[\Pi^{*}(x), \psi^{*}(x), \varphi^{*}(x), \theta^{*}(x), \sigma_{i j}^{*}(x), N^{*}(x)\right] \exp (\omega t+i b z),
$$

where $\omega$ is the (complex circular frequency) time constant, $i$ is the imaginary, $b$ be a wave number in the $z$-direction and $\Pi^{*}, \psi^{*}, N^{*}(x), \varphi^{*}(x), \theta^{*}(x)$ and $\sigma_{i j}^{*}(x)$ are the amplitude of the field functions. By using the normal mode defined in the Eq. (27), Eqs. (18)-(22), we arrive at a system of five homogeneous equations:

$$
\begin{gathered}
\left(D^{2}-\alpha_{1}\right) N^{*}+\varepsilon_{3} \theta^{*}=0, \\
\left(D^{2}-b^{2}\right) \phi^{*}-\omega \theta^{*}+\varepsilon_{2} N^{*}+\alpha_{2}\left(D^{2}-b^{2}\right) \Pi^{*}=0, \\
\left(\alpha D^{2}-\alpha_{3}\right) \Pi^{*}-\theta^{*}-N^{*}=0, \\
\left(D^{2}-\alpha_{4}^{2}\right) \psi^{*}=0, \\
\left(D^{2}-A_{1}\right) \phi^{*}+\beta^{*} \theta^{*}=0,
\end{gathered}
$$

Stress components equations (24)-(26) will take the following forms:

$$
\begin{gathered}
\sigma_{x x}^{*}=\alpha_{5} D^{2} \Pi^{*}-\alpha_{6} b^{2} \Pi^{*}+2 i b D \psi^{*}-\alpha_{5}\left(\theta^{*}+N^{*}\right)-P^{*}, \\
\sigma_{z z}^{*}=-\alpha_{5} b^{2} \Pi^{*}+\alpha_{6} D^{2} \Pi^{*}-2 i b D \psi^{*}-\alpha_{5}\left(\theta^{*}+N^{*}\right)-P^{*}, \\
\sigma_{x z}^{*}=2 i b D \Pi^{*}-\left(\alpha_{7} D^{2}+\alpha_{8} b^{2}\right) \psi^{*} .
\end{gathered}
$$

where

$$
D=\frac{d}{d x}, \quad \alpha_{1}=b^{2}+q_{1}+\omega q_{2}, \quad \alpha_{2}=\varepsilon_{1} \omega, \quad \alpha_{3}=\alpha b^{2}+R_{H} \omega^{2},
$$




$$
\alpha_{4}^{2}=b^{2}+\omega^{2} \beta^{2} R_{H}, \quad A_{1}=b^{2}+\beta^{*}, \beta^{*}=\frac{1}{a}, \alpha_{5}=\frac{(2 \mu+\lambda)}{\mu}, \quad \alpha_{6}=\frac{\lambda}{\mu}, \alpha_{7}=\frac{s_{1}}{\mu},
$$

$\alpha_{8}=\frac{s_{2}}{\mu}$,

The system of Eqs. (28), (29), (30) and (32) has non-trivial solutions if and only if the determination of the factor matrix vanishes. So

$$
\left|\begin{array}{cccc}
0 & 0 & D^{2}-\alpha_{1} & \varepsilon_{3} \\
\alpha_{2}\left(D^{2}-b^{2}\right) & \left(D^{2}-b^{2}\right) & \varepsilon_{2} & -\omega \\
\left(\alpha D^{2}-\alpha_{3}\right) & 0 & -1 & -1 \\
0 & \left(D^{2}-A\right) & 0 & \beta^{*}
\end{array}\right|=0 .
$$

We get a sixth order equation by eliminating $\theta^{*}(x), \Pi^{*}(x), N^{*}(x)$ and $\varphi^{*}(x)$ between Eqs. (28)-(30) and (32), we obtain the partial differential equation satisfied by $\theta^{*}(x)$

$$
\left[D^{6}-E D^{4}+F D^{2}-G\right] \theta^{*}(x)=0,
$$

where

$$
\begin{gathered}
\alpha_{1}^{*}=\alpha_{1}+\varepsilon_{3}, \quad \alpha_{3}^{*}=\alpha_{3} / R_{H}, \quad A_{2}=\alpha \beta^{*}+\omega\left(\alpha-\varepsilon_{1}\right), \quad \beta_{1}^{*}=\beta^{*} R_{H} \\
A_{3}=\alpha_{3}+\alpha A_{1}, \quad A_{4}=\left(\alpha_{1}^{*}+A_{1}\right), \quad \omega^{*}=\omega R_{H} \\
E=\left[\left(\alpha_{3}+\alpha\left(\alpha_{1}+b^{2}\right)\right) \beta^{*}+\left(\alpha\left(\alpha_{1}+A_{1}\right)+\alpha_{3}\right) \omega++\alpha \varepsilon_{2} \varepsilon_{3}+\left(\alpha_{1}^{*}+\varepsilon_{3}+A_{1}+b^{2}\right)\right] / A_{2}, \\
F=\left[\left(\alpha_{1} \alpha_{3}+\left(\alpha \alpha_{1}+\alpha_{3}\right) b^{2}\right) \beta^{*}+\left(\alpha_{1} A_{3}+\alpha_{3} A_{1}\right) \omega-\varepsilon_{2} \varepsilon_{3} A_{3}+\omega \varepsilon_{1}\left(\alpha_{1}^{*} A_{1}+b^{2} A_{4}\right)\right] / A_{2}, \\
G=\left[\alpha_{1} \alpha_{3} b^{2} \beta_{1}^{*}+\alpha_{1} \alpha_{3} A_{1} \omega-R_{H} \varepsilon_{2} \varepsilon_{3} \alpha_{3} A_{1}-\omega \varepsilon_{1} \alpha_{1}^{*} A_{1} b^{2}\right] / A_{2},
\end{gathered}
$$

The above equation can be factorized

$$
\left(D^{2}-k_{1}^{2}\right)\left(D^{2}-k_{2}^{2}\right)\left(D^{2}-k_{3}^{2}\right) \theta^{*}(x)=0,
$$

where, $k_{n}^{2}(n=1,2,3)$ are the roots of the following characteristic equation

$$
k^{6}-E k^{4}+F k^{2}-G=0
$$

Equation (31) together with the characteristic equation of (37) can be written as,

$$
\left(D^{2}-k_{1}^{2}\right)\left(D^{2}-k_{2}^{2}\right)\left(D^{2}-k_{3}^{2}\right)\left(D^{2}-k_{4}^{2}\right)=0 \text {, }
$$

where $k_{4}^{2}$ are the roots of equation (31).

The solution of Eq. (43) which is bounded as $x \rightarrow \infty$, is given by

$$
\theta^{*}(x)=\sum_{n=1}^{3} M_{n}(b, \omega) \exp \left(-k_{n} x\right)
$$

Similarly 


$$
\begin{gathered}
\Pi^{*}(x)=\sum_{n=1}^{3} M_{n}^{\prime}(b, \omega) \exp \left(-k_{n} x\right) \\
N^{*}(x)=\sum_{n=1}^{3} M_{n}^{\prime \prime}(b, \omega) \exp \left(-k_{n} x\right) \\
\varphi^{*}(x)=\sum_{n=1}^{3} M_{n}^{\prime \prime \prime}(b, \omega) \exp \left(-k_{n} x\right) \\
\psi^{*}(x)=M_{4}(b, \omega) \exp \left(-k_{4} x\right)
\end{gathered}
$$

since,

$$
\begin{aligned}
& u^{*}(x)=D \Pi^{*}+i b \psi^{*}, \\
& w^{*}(x)=i b \Pi^{*}-D \psi^{*},
\end{aligned}
$$

Using Eqs. (49) and (50), in order to obtain the amplitude of the displacement components $u$ and $w$, which are bounded as $x \rightarrow \infty$, then Eqs. (49) and (50) become

$$
\begin{aligned}
& u^{*}(x)=-\sum_{n=1}^{3} M_{n}^{\prime}(b, \omega) k_{n} e^{-k_{n} x}+i b M_{4}(b, \omega) \exp \left(-k_{4} x\right), \\
& w^{*}(x)=i b \sum_{n=1}^{3} M_{n}^{\prime}(b, \omega) e^{-k_{n} x}+M_{4}(b, \omega) k_{4} \exp \left(-k_{4} x\right) .
\end{aligned}
$$

Where $M_{4} M_{n}, M_{n}^{\prime}, M_{n}^{\prime \prime}$ and $M_{n}^{\prime \prime \prime}$ are some parameters depending on $b$ and $\omega$.

Substituting from Eqs. (44)-(47) into Eqs. (28)-(30) and (32), we obtain

$$
\begin{array}{cr}
M_{n}^{\prime}(b, \omega)=H_{1 n} M_{n}(b, \omega), & n=1,2,3 . \\
M_{n}^{\prime \prime}(b, \omega)=H_{2 n} M_{n}(b, \omega), & n=1,2,3, \\
M_{n}^{\prime \prime \prime}(b, \omega)=H_{3 n} M_{n}(b, \omega), & n=1,2,3 .
\end{array}
$$

where

$$
\begin{array}{cc}
H_{1 n}=\frac{\left(k_{n}^{2}-\alpha_{1}^{*}\right)}{\left(k_{n}^{2}-\alpha_{1}\right)\left(\alpha k_{n}^{2}-\alpha_{3}\right)}, & n=1,2,3 . \\
H_{2 n}=-\frac{\varepsilon_{3}}{\left(k_{n}^{2}-\alpha_{1}\right)}, & n=1,2,3 . \\
H_{3 n}=-\frac{\beta^{*}}{k_{n}^{2}-A_{1}}, & n=1,2,3 .
\end{array}
$$

Thus, we have 


$$
\begin{gathered}
\Pi^{*}(x)=\sum_{n=1}^{3} H_{1 n} M_{n}(b, \omega) \exp \left(-k_{n} x\right) \\
N^{*}(x)=\sum_{n=1}^{3} H_{2 n} M_{n}(b, \omega) \exp \left(-k_{n} x\right) \\
\varphi^{*}(x)=\sum_{n=1}^{3} H_{3 n} M_{n}(b, \omega) \exp \left(-k_{n} x\right)
\end{gathered}
$$

Substitution from Eqs. (44), (48) and (49)- (51) into Eqs. (33)- (35), we get

$$
\begin{gathered}
\sigma_{x x}^{*}=\sum_{n=1}^{3} h_{n} M_{n}(b, \omega) \exp \left(-k_{n} x\right)-2 i b k_{4} M_{4} \exp \left(-k_{4} x\right)-P^{*} \\
\sigma_{z z}^{*}=\sum_{n=1}^{3} h_{n}^{\prime} M_{n}(b, \omega) \exp \left(k_{n} x\right)+2 i b k_{4} M_{4} \exp \left(k_{4} x\right)-P^{*} \\
\sigma_{x z}^{*}=\sum_{n=1}^{3} h_{n}^{\prime \prime} M_{n}(b, \omega) \exp \left(-k_{n} x\right)-\left(\alpha_{7} k_{4}^{2}+\alpha_{8} b^{2}\right) M_{4} \exp \left(-k_{4} x\right) \\
u^{*}(x)=-\sum_{n=1}^{3} k_{n} H_{1 n} M_{n}(b, \omega) e^{-k_{n} x}+i b M_{4} e^{-k_{4} x} \\
w^{*}(x)=\sum_{n=1}^{3} i b H_{1 n} M_{n}(b, \omega) e^{-k_{n} x}+k_{4} M_{4} e^{-k_{4} x}
\end{gathered}
$$

where

$$
\begin{aligned}
& h_{n}=H_{1 n}\left(\alpha_{5} k_{n}^{2}-\alpha_{6} b^{2}\right)-\alpha_{5}\left(1+H_{2 n}\right) \\
& h_{n}^{\prime}=-H_{1 n}\left(\alpha_{5} b^{2}-\alpha_{6} k_{n}^{2}\right)-\alpha_{5}\left(1+H_{2 n}\right) \\
& h_{n}^{\prime \prime}=-2 i b k_{n}
\end{aligned}
$$

\section{Applications}

In this section, we determine the parameters $M_{n}(n=1,2,3,4)$. In the physical problem, we should suppress the positive exponentials that are unbounded at infinity. The constants $M_{1}, M_{2}, M_{3}, M_{4}$ have to chosen such that the boundary conditions on the surface at $x=0$ (suppose the boundary $x=0$ is adjacent to vacuum) take the form:

i) Mechanical boundary condition that surface of the half-space is traction load

$$
\sigma_{x x}(0, z, t)=-p_{1} \exp (\omega t+i b z)
$$

ii) Mechanical boundary condition that surface of the half-space is traction free

$$
\sigma_{x z}(0, z, t)=0
$$

iii) Assuming that the boundary $\quad x=0$ is thermally insulated, we have 


$$
\frac{\partial T(0, z, t)}{\partial x}=0
$$

vi) During the diffusion process, the carriers can reach the sample surface, with a finite probability of recombination. So the boundary condition for the carrier density can be given below:

$$
\frac{\partial N(0, z, t)}{\partial x}=\frac{s}{D_{e}} N
$$

Substituting the expressions of the variables considered into the above boundary conditions, we can obtain the following equations satisfied by the parameters

$$
\begin{gathered}
\sum_{n=1}^{3} h_{n} M_{n}(b, \omega)-2 i b k_{4} M_{4}-P=-p_{1} \\
\sum_{n=1}^{3} h_{n}^{\prime \prime} M_{n}(b, \omega)-\left(\alpha_{7} k_{4}^{2}+\alpha_{8} b^{2}\right) M_{4}=0 \\
\sum_{n=1}^{3} k_{n} M_{n}(b, \omega)=0 \\
\sum_{n=1}^{3} k_{n} H_{2 n} M_{n}(b, \omega)=-\frac{s}{D_{e}} N
\end{gathered}
$$

Invoking the boundary conditions (63)-(65) at the surface $\mathrm{X}=0$ of the plate, we obtain a system of four equations. After applying the inverse of matrix method (or Cramer's rule), we have the values of the four constants $M_{j}, j=1,2,3,4$. Hence, we obtain the expressions of displacements, temperature distribution, Carrier density and another physical quantity of the plate.

\section{Numerical results and discussions}

In order to analyze the above problem numerically, we now consider a numerical example for which computational results are given. The silicon (Si) is chosen as the material for numerical simulation. The numerical constants (parameters in SI unit) of the problem are taken as:

\begin{tabular}{|l|l|l|l|}
\hline$\lambda=3.64 \times 10^{10} \mathrm{~N} / \mathrm{m}^{2}$ & $\mu=5.46 \times 10^{10} \mathrm{~N} / \mathrm{m}^{2}$ & $\rho=2330 \mathrm{~kg} / \mathrm{m}^{3}$ & $\tau=5 \times 10^{-5} \mathrm{~s}$ \\
\hline$T_{0}=800 \mathrm{~K}$ & $d_{n}=-9 \times 10^{-31} \mathrm{~m}^{3}$ & $D_{E}=2.5 \times 10^{-3} \mathrm{~m}^{2} / \mathrm{s}$ & $E_{g}=1.11 \mathrm{eV}$ \\
\hline$C_{e}=695 \mathrm{~J} /(\mathrm{kg} \mathrm{K})$ & $\alpha_{t}=4.14 \times 10^{-6} \mathrm{~K}^{-1}$ & $s=2 \mathrm{~m} / \mathrm{s}$ & $t=0.8$ \\
\hline$\omega_{0}=-0.3$ & $P=10$ & $\xi=0.1$ & $z=1$ \\
\hline$b=1$ & $p_{1}=2$ & $\mu_{0}=4 \pi \times 10^{-7} \mathrm{H} / \mathrm{m}$ & $\varepsilon_{0}=8.85 \times 10^{-12} \mathrm{~F} / \mathrm{m}$ \\
\hline
\end{tabular}

Since we have $\omega=\omega_{0}+i \xi$ where the imaginary unit is $i, e^{\omega t}=e^{\omega_{0} t}(\cos \xi t+i \sin \xi t)$

and for small value of time, we can take $\omega=\omega_{0}$ (real). The computations were carried out for a value of time $t=0.7$. The numerical technique, outlined above, was used for the distribution of the real part of the thermal temperature $\theta$, the displacement components $u$, Carrier density $\mathrm{N}$ and the stress $\left(\sigma_{x x}, \sigma_{x z}\right)$ distribution for the problem. Here all the variables are taken in non dimensional forms and displayed graphically as 2D and 3D form in Figs. 1-6.

\subsection{Effect of magnetic field}


Figure 1 shows the positive influence of magnetic field $H_{0}$ on non-dimensional temperature distribution, displacement distribution, normal force distribution, force stress distribution and carrier density, respectively with respect to axis $\mathrm{x}$. the different values of magnetic field are shown by solid $\left(H_{0}=0.0\right)$, dashed $\left(H_{0}=10\right)$ and dash-dot $\left(H_{0}=20\right)$ lines respectively when $\varepsilon_{3}=1.8$ and hydrostatic initial stress $P=10$. The values of temperature for a non-magnetic thermoelastic medium under the influence of initial stress decrease sharply in the beginning and smooth increases to arrive the maximum value and then oscillate uniformly. The variations of temperature are similar in nature for both differences in magnitude of magnetic field. The heat conduction and the dynamical heat take the same behavior. Under the influence of magnetic field, the values of normal force stress first increases in the first range and then oscillate uniformly. Also the values of shear force stress for a thermoelastic medium without magnetic field lie in a very short range and have a very similar to the variation of normal force stress with difference in different values of magnetic field. The Carrier density start from its minimum value, increase with an increasing of $x$ tend to zero as $\mathrm{x}$ tends to infinity but $u$ starts from maximum, decreases with an increasing of axis $x$ tends to zero as $x$ tends to infinity, this indicate to the vanishing of all components for the large values of $x$.

We obtain from this figure, that the effect of magnetic field caused rearranged the atoms in the medium and compact the curves when $x$ increases.

\subsection{Effect of thermoelectric coupling parameter}

Figure 2 plots the positive influence of thermoelectric coupling parameter $\varepsilon_{3}$ on the temperature distribution, displacement distribution, normal force distribution, force stress distribution, Carrier density $N$ and the heat conduction $\phi$, respectively with respect to axis $x$. It is obvious that $T, \phi$ and $N$ start from its minimum value, increase with an increasing of $x$ tend to zero as $x$ tends to infinity but $\sigma_{x z}$ and $\sigma_{x x}$ start from its primary values, decreases with an increasing of axis $x$ tends to zero as $x$ tends to infinity, this indicate to the vanishing of all components for the large values of $x$, also, it is obvious that $\sigma_{x x}$ and $\sigma_{x z}$ start from zero at $x=0$ this indicate that there is no stresses in the origin value of $x$. From this figure, we can obtain significant effect of thermoelectric coupling parameter in the range.

\subsection{Effect of two temperature parameter}

In Figure. 3 some comparisons have been shown to estimate the effect of two-temperature parameters $\mathrm{a}=\{0,5\}$ on the temperature distribution, displacement distribution, normal force distribution, force stress distribution, Carrier density $N$, respectively, with respect to axis $x$, which agrees the initial boundary conditions. It is also observed from these figure that with the increase of dimensionless two-temperature parameter leads to an increase in its magnitude for all physical quantities. It is concluded that the temperature $\mathrm{T}$ and shear stress $\sigma_{x z}$ influences positively without two temperature parameters (i.e., $\mathrm{T}$ takes higher values if $\mathrm{a}=5$ comparing with the corresponding value $\mathrm{a}=0$ ) but vice versa concerns $\sigma_{x x}$. Also, it is shown from the shape of the displacement $u$ takes higher values without the two temperature parameters with the small values of $x$ and takes inverse behavior with the large values of $x$ comparing with the corresponding values of $u$ if there is no two temperature parameter.

\subsection{Effect of hydrostatic initial stress}

Figure. 4 shows the comparison between the temperature T, $\phi$, displacement components $\mathrm{u}$, , the force stresses components $\sigma_{x x}, \sigma_{x z}$ and the Carrier density $N$, the case of different two values of hydrostatic initial stress, (namely $P=0.0$ and $P=100$ ) with magnetic field. It is clear from the graph that most of the physical quantities have minimum value at the beginning at $x=0$, it 
begins to fall just near the edge ( $x=0$ ), where it experiences sharp increases (with minimum negative gradient). In addition, all lines begin to intersect when the horizontal distance $x$ is large to reach the reference temperature of the solid. These results obey physical reality for the behaviour of silicon as a polycrystalline solid (state of particles equillibrium). In addition, all lines begin to coincide when the horizontal distance $x$ tends to infinity and reach zero after their relaxations at infinity.

\subsection{Effect of all parameters in $3 D$}

Figures. 5 and 6 clears the 3D schematics concern the temperature distribution $T, \phi$, displacement distribution $u, w$, stresses force distribution and Carrier density $N$ with respect to $x$ and $z$ axes in the presentce of magnetic field and the hydrostatic initial stress with thermoelectric coupling parameter $\varepsilon_{3}$ and thermoelastice coupling parameter $\varepsilon_{1}$. It is obvious that all values increase from its primary values at $x=0$ and decrease tends to zero as $x$ tends to infinity. We obtain for all physical quantities, The Elastic Wave Motions in 3D overlapping and damping when $x$ and $z$ increases to reach the state of particles equillibrium. These figures are very important to study the dependence of these physical quantities on the vertical component of distance. The curves obtained are highly depending on the vertical distance from origin, all the physical quantities are moving in wave propagation.

\section{Conclusion}

Using the harmonic wave analysis method, we obtained an explicit, totally analytic, uniformly valid solution of the system of four fully coupled, highly linear similarity equations describing heat and mass transfer by mixed convection in homogeneous and isotropic properties of the medium with two temperatures. We can conclude that the magnetic field has a great effect on the temperature distribution, displacement components, normal force distribution and force stress distribution and this effect produces the same trend under the effect of photothermal theory. All functions tend to zero as $x$-axis tends to infinity, indicate to the vanishing of all components for the large values of $x$. The two temperature generalized theory of magneto-thermo-elasticity describes the behavior of the particles of an elastic body is more realistic than the one temperature theory of generalized magnetothermo-elasticity. Positive thermoelectric couple parameter, it appears that the positive value has a good influence on all parameters. Finally, it is clear from the results obtained numerically and graphically, that photothermal has a good influence on the phenomena which has a lot of applications on diverse field as semiconducting and the reactions during a photothermal process and other fields in physical engineering also in physical chemistry and medical physics.

\section{References}

[1]M.A. Biot, Thermoclasticity and irreversible thermodynamics. J. Appl. Phys. 27, 240-253 (1956).

[2]H. Lord, Y. Shulman, A generalized dynamical theory of thermoelasticity. J. Mech. Phys. Solids 15, 299-309 (1967).

[3] A.E. Green, K.A. Lindsay, Thermoelasticity. J. Elast. 2, 1-7 (1972) .

[4]D.S. Chandrasekharaiah, Thermoelasticity with second sound: a review. Appl. Mech. Rev. 39, 355-376 (1986)

[5]D.S. Chandrasekharaiah, Hyperbolic thermoelasicity: a review of recent literature. Appl. Mech. Rev. 51, 705729 (1998)

[6]J.N. Sharma, V. Kumar, C. Dayal, Reflection of generalized thermoelastic waves from the boundary of a halfspace. J. Therm. Stresses 26, 925-942 (2003)

[7]J.P. Gordon, R.C.C. Leite, R.S. Moore, S.P.S. Porto, J.R. Whinnery, Long- $\quad$ transient effects in lasers with inserted liquid samples. Bull. Am. Phys. Soc. 119, 501 (1964).

[8]L.B. Kreuzer, Ultralow gas concentration infrared absorption spectroscopy. J. Appl. Phys. 42, 2934 (1971).

[9] A.C. Tam, Ultrasensitive Laser Spectroscopy, pp. 1-108. Academic Press, New York (1983).

[10]A.C. Tam, Applications of photoacoustic sensing techniques. Rev. Mod. Phys. 58, 381 (1986).

[11]A.C. Tam, Photothermal Investigations in Solids and Fluids, pp. 1-33. Academic Press, Boston (1989) 
[12]D.M. Todorovic, P.M. Nikolic, A.I. Bojicic, Photoacoustic frequency transmission technique: electronic deformation mechanism in semiconductors. J. Appl. Phys. 85, 7716 (1999).

[13] P. Puri, Plane waves in thermoelasticity and magneto- thermoelasticity. Int. J. Eng.Sci., vol. 10, pp. 467-476, 1972.

[14] A. Nayfeh and S. Nemat-Nasser, Transient thermoelastic waves in half-space with thermal relaxation. ZAMP., vol. 23, pp. 52-68, 1972.

[15]P.J. Chen, M.E. Gurtin, W.O. Williams, On the thermodynamics of non- simple elastic materials with two temperatures. Zamp 20, 107-112 (1969).

[16]J.K. Chen, J.E. Beraun, C.L. Tham, Ultrafast thermoelasticity for short-pulse laser heating. Int. J. Eng. Sci. 42, 793-807 (2004).

[17]T.Q. Quintanilla , C. L. Tien, Heat transfer mechanism during short-pulse laser heating of metals, ASME J. Heat Transfer 115, 835-841 (1993).

[18]H.M. Youssef, Theory of two-temperature-generalized thermoelasticity, IMA Journal of Applied Mathematics 71, 383-390 (2006).

[19]M.I.A. Othman, Kh. Lotfy, On the plane waves of generalized thermo-microstretch elastic half-space under three theories, Int., Comm., in Heat and Mass Transfer 37, 192 (2010).

[20]M.I.A. Othman, S.M. Abo-dahab, Kh. Lotfy Gravitational effect and initial stress on generalized magnetothermo-microstretch elastic solid for different theories, App. Math. \& Comp. 230, 597-615 (2014).

[21]Kh. Lotfy, Two temperature generalized magneto-thermoelastic interactions In an elastic medium under three theories, App., Math., and Comp. 227, 871-888 (2014).

[22]Kh. Lotfy, W. Hassan, Normal mode method for two-temperature generalized thermoelasticity under thermal shock problem, Journal of Thermal Stresses, 37(5), 545-560 (2014).

[23] Kh. Lotfy and S.M. Abo-Dahab, Generalized magneto-thermoelasticity with fractional derivative heat transfer for a rotation of a fibre- reinforced thermoelastic, J. Comp. Theo. Nanoscience 12(8), 1869-1881 (2015).

[24] A. Mandelis, M. Nestoros, C. Christofides, Thermoelectronic-wave coupling in laser photothermal theory of semiconductors at elevated temperature, Opt. Eng. 36, 459 (1997).

[25] D.M. Todorovic, Plasma, thermal, and elastic waves in semiconductors, Rev. Sci. Instrum. 74, 582 (2003).

[26]A.N. Vasil'ev, V.B. Sandomirskii, Photoacoustic effects in finite semiconductors, Sov. Phys. Semicond. 18, 1095 (1984).

[27]C. Christofides, A. Othonos, Loizidou, E.: Influence of temperature and modulation frequency on the thermal activation coupling term in laser photothermal theory, J. Appl. Phys. 92, 1280 (2002).

[28] Y.Q. Song, J.T. Bai, Z.Y. Ren, Study on the reflection of photothermal waves in a semiconducting medium under generalized thermoelastic theory, Acta Mech. 223, 1545 (2012). 

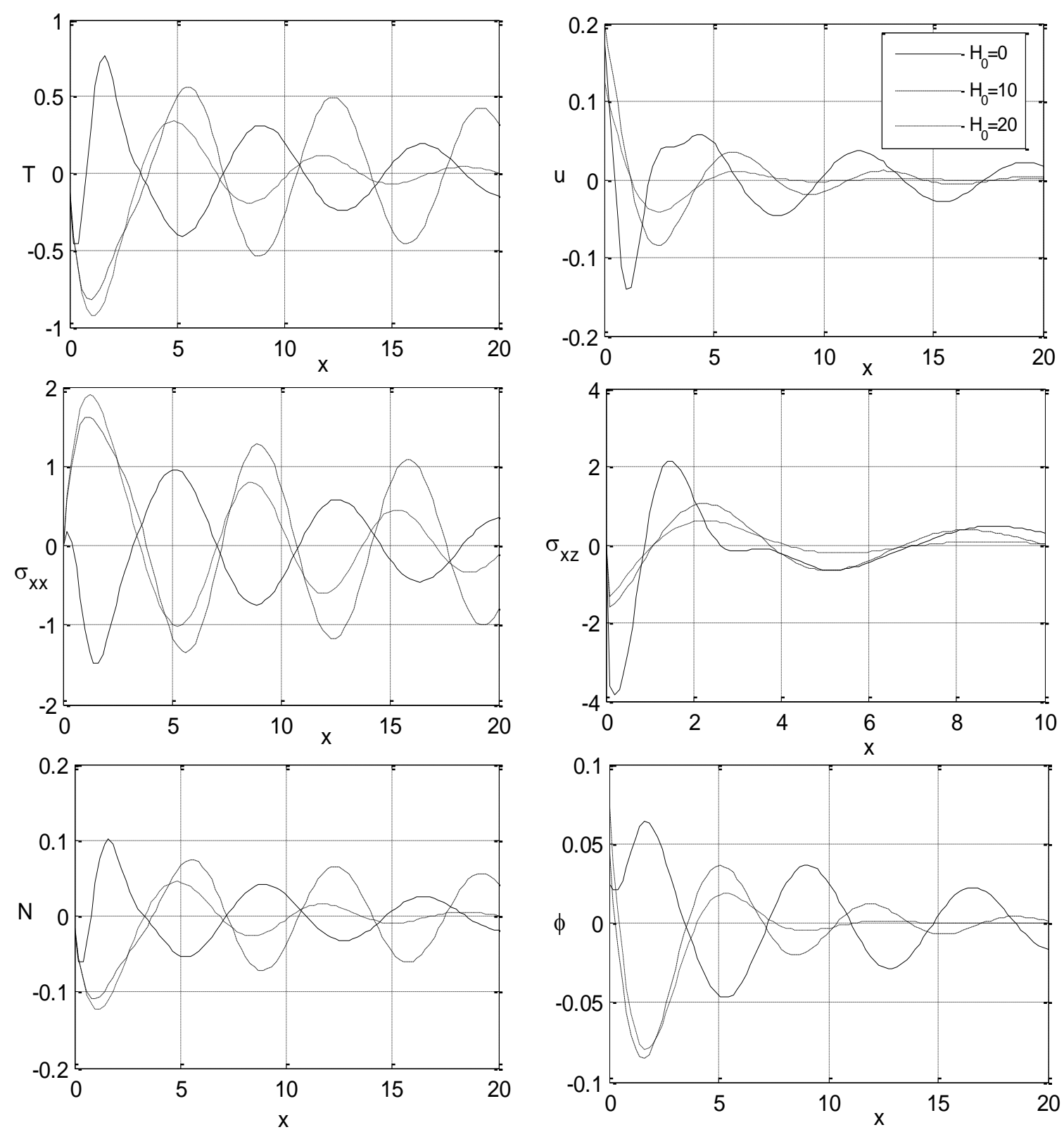

Figure 1 Variation of physical quantities at different values of magnetic field. 

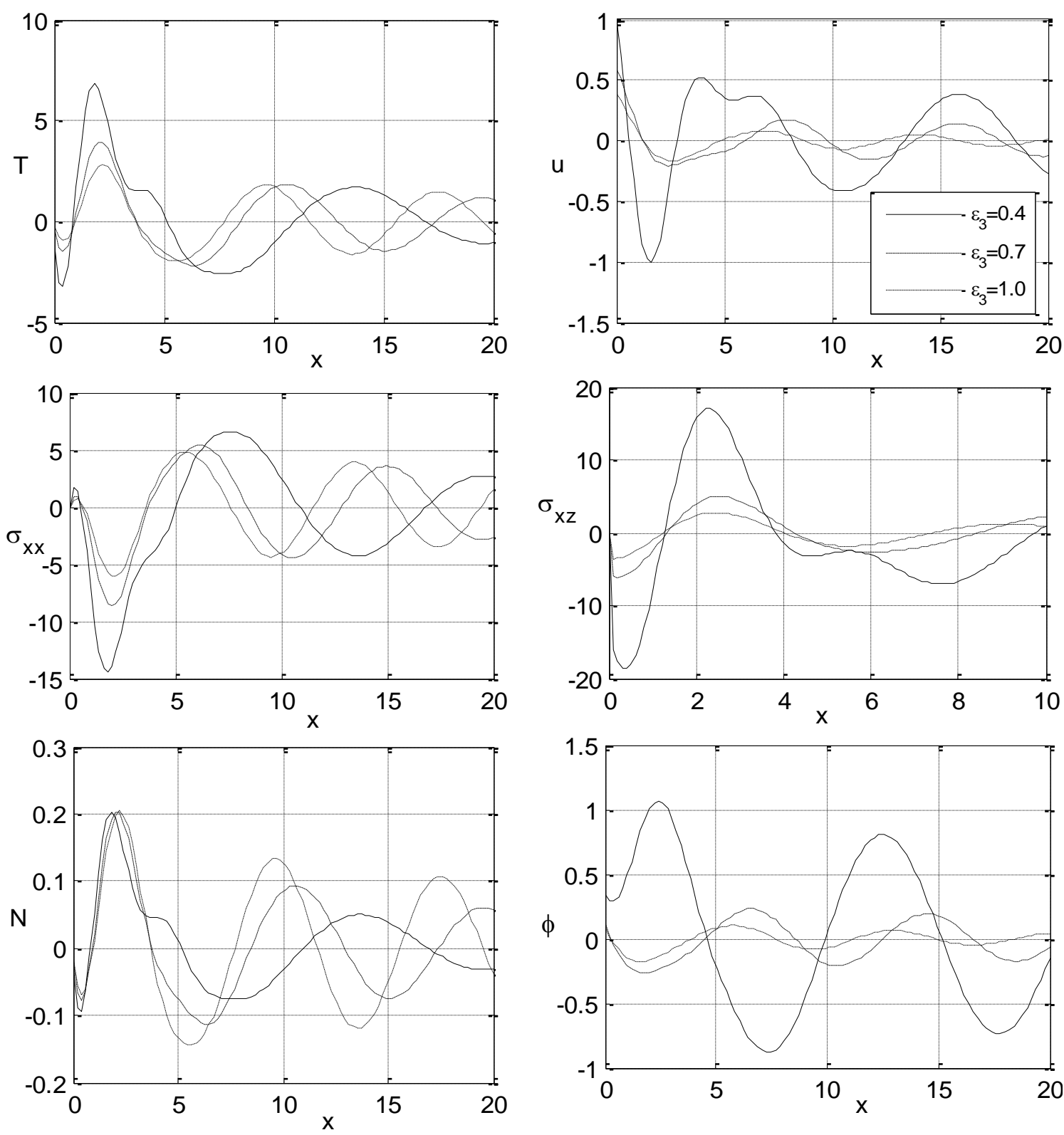

Figure 2 Variation of physical quantities at different values of positive thermoelectric coupling parameter with two temperature when $H_{0}=10$. 

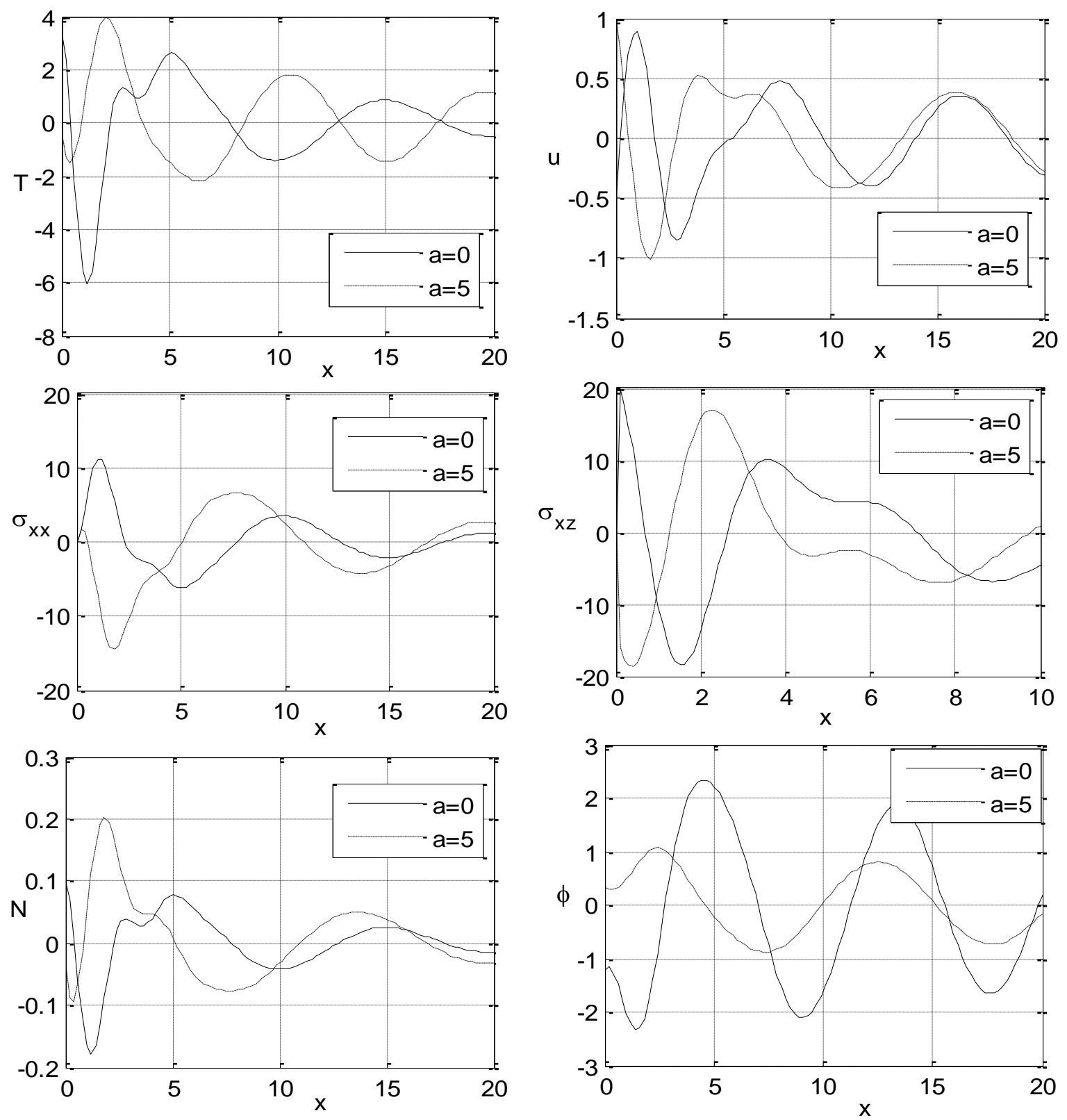

Figure 3 Variation of physical quantities at different values of two temperature with thermoelectric coupling parameter at $H_{0}=10$ under the hydrostatic initial stress 

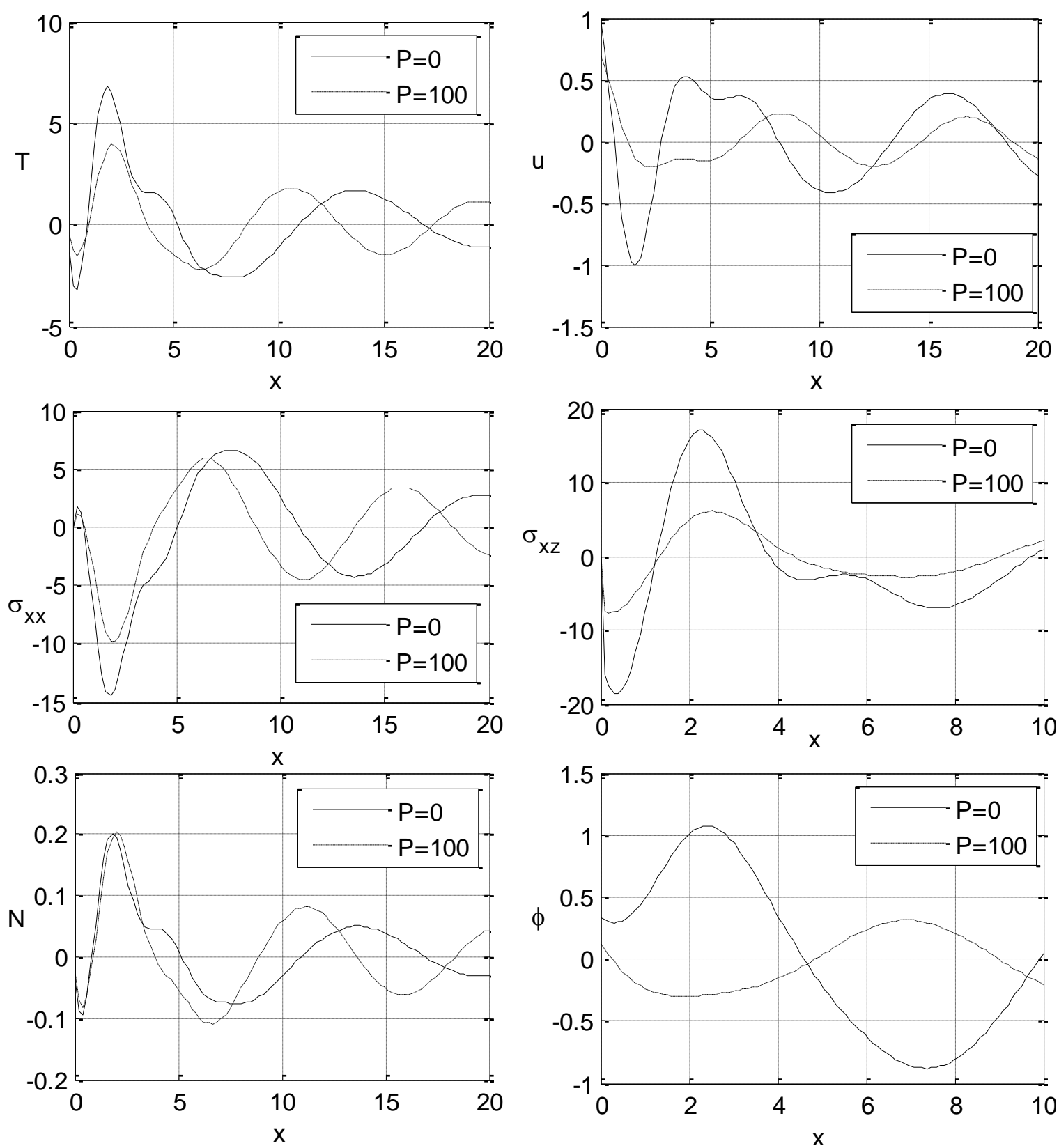

Figure 4 Variation of physical quantities at different values of the hydrostatic initial stress under two temperature effect with thermoelectric coupling parameter and $H_{0}=10$. 

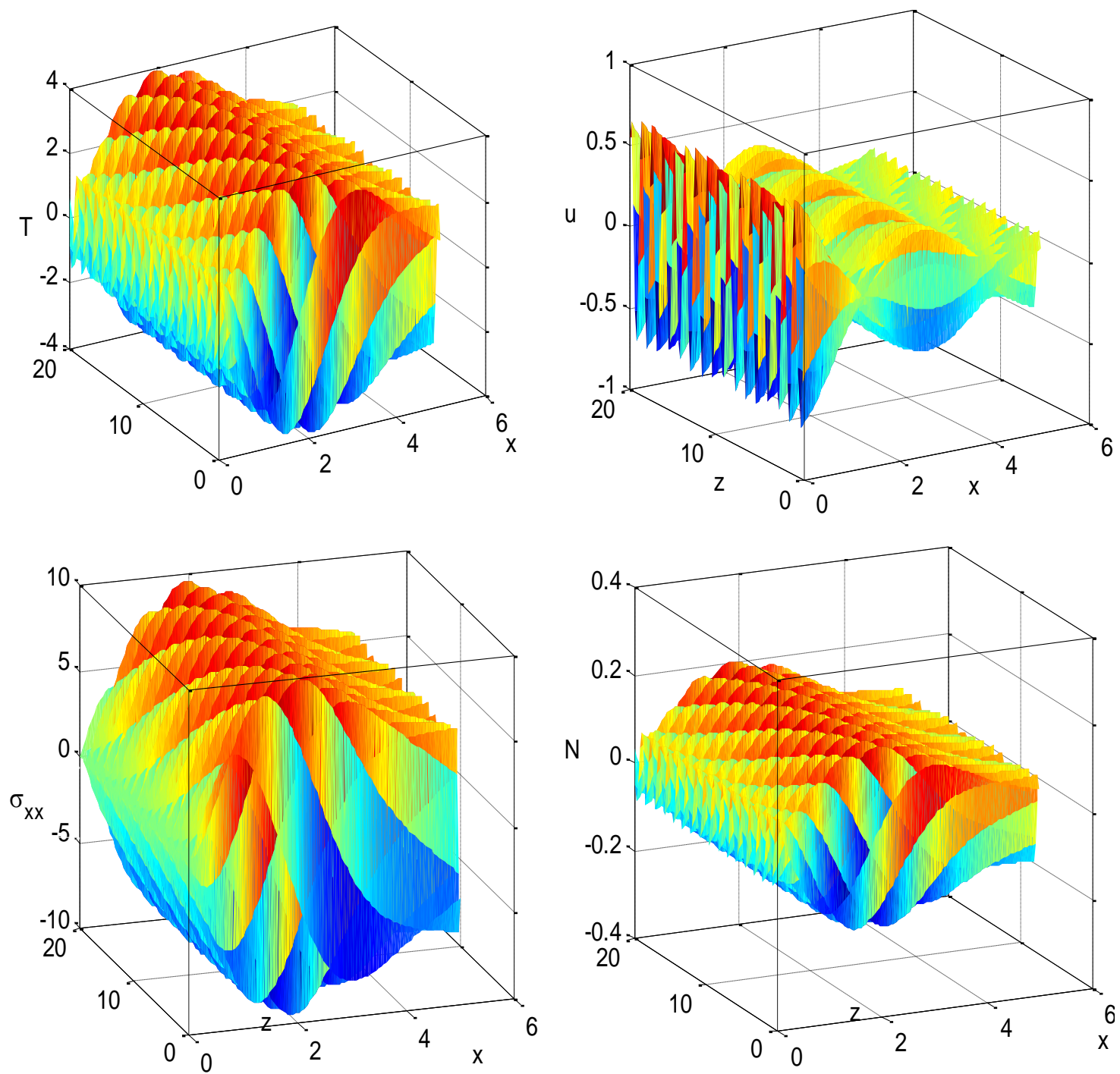

Figure 5 some physical quantities in $3 \mathrm{D}$ against $\mathrm{x}$ and $\mathrm{z}$ under the influence of the hydrostatic initial stress, two temperature parameter, thermoelectric coupling parameter and $H_{0}=10$. 

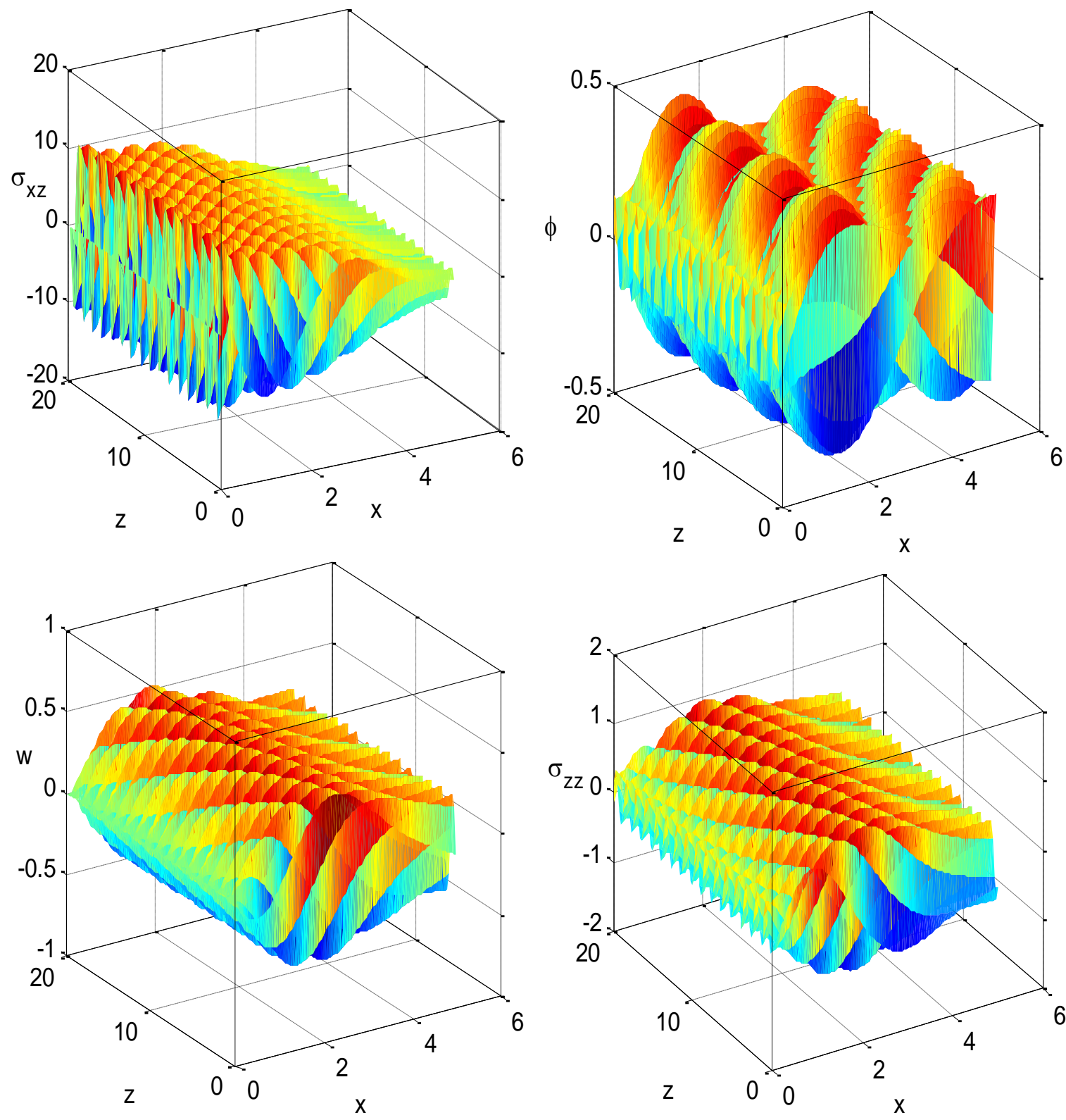

Figure 6 some physical quantities in $3 \mathrm{D}$ against $\mathrm{x}$ and $\mathrm{z}$ under the influence of the hydrostatic initial stress, two temperature parameter, thermoelectric coupling parameter and $H_{0}=10$.

(C) 2016 by the authors; licensee Preprints.org, MDPI, Basel, Switzerland. This article is an open access article distributed under the terms and conditions of the Creative Commons by Attribution (CC-BY) license (http://creativecommons.org/licenses/by/4.0/). 